\section{A Closed-Form Expression for the Uncertainty in Odometry Position Estimate of an Autonomous Vehicle}

Josep M. Mirats Tur, José Luis Gordillo, and Carlos Albores Borja

\begin{abstract}
Using internal and external sensors to provide position estimates in a two-dimensional space is necessary to solve the localization and navigation problems for a robot or an autonomous vehicle (AV). Usually, a unique source of position information is not enough, so researchers try to fuse data from different sensors using several methods, as, for example, Kalman filtering. Those methods need an estimation of the uncertainty in the position estimates obtained from the sensory system. This uncertainty is expressed by a covariance matrix, which is usually obtained from experimental data, assuming, by the nature of this matrix, general and unconstrained motion. We propose in this paper a closed-form expression for the uncertainty in the odometry position estimate of a mobile vehicle, using a covariance matrix whose form is derived from the cinematic model. We then particularize for a nonholonomic Ackerman driving-type AV. Its cinematic model relates the two measures being obtained for internal sensors: the velocity, translated into the instantaneous displacement; and the instantaneous steering angle. The proposed method is validated experimentally, and compared against Kalman filtering.
\end{abstract}

Index Terms-Localization, navigation, nonholonomic constraints, robot positioning uncertainty.

\section{INTRODUCTION}

Mobile robots and autonomous vehicles (AVs) can assist or even substitute for humans, to tackle tedious, monotonous, and dangerous activities, such as in mining, civil engineering construction, rescue in catastrophes, or agricultural labor. To reliably perform a task while navigating in the real world, it is necessary to locate those vehicles to know their position, as well as its associated uncertainty. Usually, to accurately provide a position estimate, a unique source of position information is not enough, so researchers try to fuse data from different sources. For instance, in [1]-[3], indoor localization is performed using data from different sensors, [4]-[6] tackle self-localization and navigation, or for outdoor applications, [7] studies a Kalman-based sensor data fusion algorithm for a robot campus tour guide, and [8] and [9] use data fusion for agriculture and mining, respectively. When fusing information, Kalman filtering is frequently used with the problem of determining the covariance matrices associated with the position uncertainty, since the errors of the sensory system affect the position estimate [10], [11]. These matrices are determined experimentally from data, and the algorithms used are very sensitive to this parameter determination. Furthermore, the computation of those matrices assumes general and unconstrained motions, ignoring the nature of phenomena, as when the movement is constrained by nonholonomic architecture, leading to poor position uncertainty estimation.

Manuscript received November 2, 2004; revised March 2, 2005. This paper was recommended for publication by Associate Editor D. Sun and Editor S. Hutchinson upon evaluation of the reviewers' comments. This work was supported in part by Consejo Nacional de Ciencia y Tecnología (CONACyT) under Grant 35396, in part by the Franco-Mexican Laboratory for Informatics (LaFMI), and in part by the Spanish Superior Council for Scientific Research (CSIC).

J. M. Mirats Tur is with the Institut de Robòtica (UPC-CSIC), 08028 Barcelona, Spain (e-mail: jmirats@iri.upc.edu).

J. L. Gordillo and C. Albores Borja are with the Center for Intelligent Systems (CSI), Tecnológico de Monterrey, 64849 Monterrey N.L., México (e-mail: JLGordillo@itesm.mx; carlos.albores@itesm.mx).

Digital Object Identifier 10.1109/TRO.2005.852262
Different methods have been proposed in the literature to estimate the position uncertainty. For example, [12] employed a min/max errorbound approach, and [13] used a scalar as positon uncertainty measure, without reference to the orientation error. Uncertainty expressed by a covariance matrix is used in more recent works, such as [14]-[17]. In [18], a method for determining this covariance matrix is given for the special case of circular arc motion with constant radius of curvature. In [19], the odometry error is modeled for a synchronous-drive system, depending on parameters characterizing both systematic and nonsystematic components. None of these methods give a close and general way to compute the robot's position uncertainty. Their main limitation is, again, to consider general and unconstrained motion, ignoring possible motion constraints due to the architecture of the vehicle.

This paper proposes a method to compute a closed-form expression for the uncertainty in the position estimate obtained from internal sensors. The position uncertainty will be expressed using a covariance matrix, and determined from the position-estimation equations given by the cinematic model. So, the proposed method is general for any platform and set of sensors, taking directly into account the physical structure of the vehicle, which is translated into the control variables being manipulated and sensed. The next sections are devoted to the general description of the method and its application to a particular AV used for mining operations. For this vehicle, a brief description of its cinematic model and the required mathematical development to derive the closed-form expression for the covariance matrix representing the position estimate uncertainty are given. Finally, the proposed method was compared against the standard extended Kalman filter (EKF) in the computation of the uncertainty associated with the odometry position estimate.

\section{Position Estimate UnCERTAINTY}

Consider we are dealing with a mobile robot or AV for which a cinematic model $M(x, y, \theta, r, s, t)$ is given. Model $M$ is generally nonlinear on $x, y, \theta$ (position of the vehicle on the plane), $r$ (physical parameters of the vehicle), vector $s$ (internal sensor measurements), and $t$ (discrete time moments). We are concerned with the problem of determining the uncertainty associated with the robot's position computed from its internal sensors using the cinematic model.

Suppose that for time $t-1$ (we are using $t-1$, for $t-\Delta t, t-2$ for $t-2 \Delta t$, and so on), the position of the vehicle and its associated uncertainty $P(t-1)=[x(t-1), y(t-1), \theta(t-1)], \operatorname{Cov}\left[P_{t-1}\right]$ are known. For time $t$, after the vehicle has performed a certain movement, and sensors on the robot have noisily measured it, the new position can be obtained using $P(t)=P(t-1)+\Delta P(t)$. The position increment will be computed from the cinematic model and actual sensor measurements. Temporal variations on the physical parameters of the robot, and different sensor measurements for $\Delta x, \Delta y$, and $\Delta \theta$ are allowed, so as to maintain generality in the model (see Fig. 1). $f, g$, and $h$ represent general functions that model these increments for components $x, y$, and $\theta$, respectively

$\Delta P_{t}=\left(\begin{array}{c}\Delta x_{t} \\ \Delta y_{t} \\ \Delta \theta_{t}\end{array}\right)=\left(\begin{array}{l}f(s(t), r(t), x(t-1), y(t-1), \theta(t-1)) \\ g(s(t), r(t), x(t-1), y(t-1), \theta(t-1)) \\ h(s(t), r(t), x(t-1), y(t-1), \theta(t-1))\end{array}\right)$

Now, the uncertainty in the robot's position will depend on model inaccuracies, noise in the sensor measurements, and additive errors from 


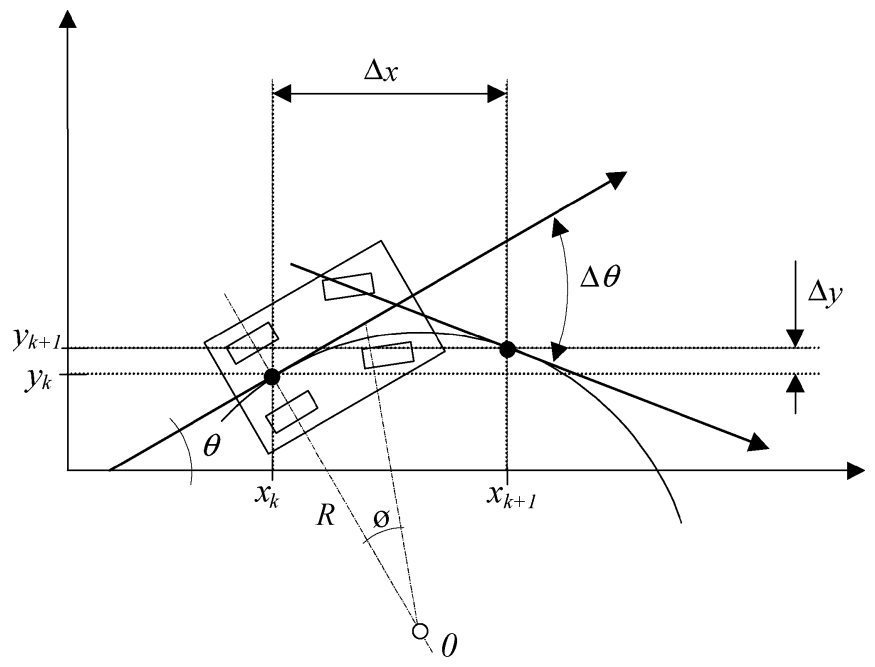

Fig. 1. Geometric diagram for the computation of $\Delta x, \Delta y$, and $\Delta \theta$. A vector with an inclination of $\theta_{t-1}+\Delta \theta / 2$ is used.

the previous position estimate. It can be computed from the covariance matrix of the robot's position

$$
\begin{aligned}
\operatorname{Cov}\left[P_{t}\right] & =\operatorname{Cov}\left[P_{t-1}+\Delta P_{t}\right] \\
& =\operatorname{Cov}\left[P_{t-1}\right]+\operatorname{Cov}\left[\Delta P_{t}\right]+2 \operatorname{Cov}\left[P_{t-1}, \Delta P_{t}\right]
\end{aligned}
$$

The term $\operatorname{Cov}\left[P_{t-1}\right]$ is recursive, and can be initialized to $0_{3 \times 3}$ if the initial position of the robot is well known. We will consider, in a first approach, that the influence of the previous position $P_{t-1}$ on the increment of run path $\Delta P_{t}$, is not meaningful. The term of interest is $\operatorname{Cov}\left[\Delta P_{t}\right]$

$$
\begin{aligned}
& \operatorname{Cov}\left[\Delta P_{t}\right] \\
& =E\left[\Delta P_{t} \Delta P_{t}^{T}\right]-E\left[\Delta P_{t}\right] E\left[\Delta P_{t}^{T}\right] \\
& E\left[\Delta P_{t} \Delta P_{t}^{T}\right] \\
& =\left(\begin{array}{lll}
E\left[\Delta x_{t} \Delta x_{t}^{T}\right] & E\left[\Delta x_{t} \Delta y_{t}^{T}\right] & E\left[\Delta x_{t} \Delta \theta_{t}^{T}\right] \\
E\left[\Delta y_{t} \Delta x_{t}^{T}\right] & E\left[\Delta y_{t} \Delta y_{t}^{T}\right] & E\left[\Delta y_{t} \Delta \theta_{t}^{T}\right] \\
E\left[\Delta \theta_{t} \Delta x_{t}^{T}\right] & E\left[\Delta \theta_{t} \Delta y_{t}^{T}\right] & E\left[\Delta \theta_{t} \Delta \theta_{t}^{T}\right]
\end{array}\right) \\
& E\left[\Delta P_{t}\right] E\left[\Delta P_{t}^{T}\right] \\
& =\left(\begin{array}{lll}
E\left[\Delta x_{t}\right] E\left[\Delta x_{t}^{T}\right] & E\left[\Delta x_{t}\right] E\left[\Delta y_{t}^{T}\right] & E\left[\Delta x_{t}\right] E\left[\Delta \theta_{t}^{T}\right] \\
E\left[\Delta y_{t}\right] E\left[\Delta x_{t}^{T}\right] & E\left[\Delta y_{t}\right] E\left[\Delta y_{t}^{T}\right] & E\left[\Delta y_{t}\right] E\left[\Delta \theta_{t}^{T}\right] \\
E\left[\Delta \theta_{t}\right] E\left[\Delta x_{t}^{T}\right] & E\left[\Delta \theta_{t}\right] E\left[\Delta y_{t}^{T}\right] & E\left[\Delta \theta_{t}\right] E\left[\Delta \theta_{t}^{T}\right]
\end{array}\right) .
\end{aligned}
$$

Equations (3)-(5) express the uncertainty of the position determined from internal sensors, while taking into account the physical architecture of the vehicle. In order to solve (4) and (5), a distribution for the sensors noise must be assumed, which will be determined from experimental characterization.

\section{RoBot PlATFORM USED}

A standard vehicle for mining operations has been used in this study. Manufactured by Johnson Industries (Pikeville, KY), the vehicle is electrically powered and manually driven. Direction, as well as driving wheels, were automated, in order to perform mine material transport

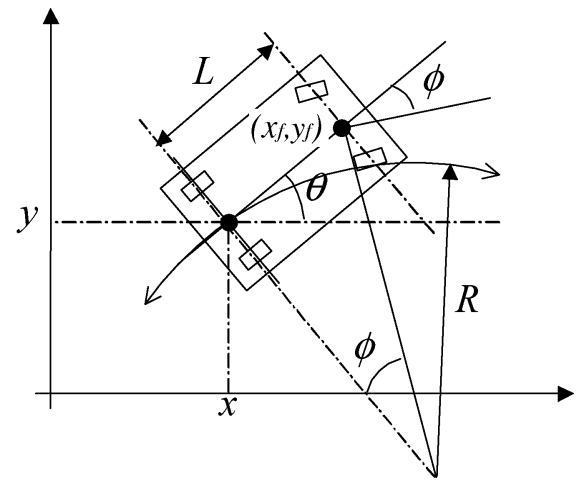

Fig. 2. Cinematic model for a common car-like vehicle.

operations outside the mine. Considering the bicycle model, the cinematic model of the AV being used [20] appears in Fig. 2. There are two nonholonomic constraints, one for the steer wheel, and the other for the rear wheel

$$
\dot{x}_{f} \sin (\theta+\phi)-\dot{y}_{f} \cos (\theta+\phi)=0, \quad \dot{x} \sin \theta-\dot{y} \cos \theta=0
$$

where $\left(x_{f}, y_{f}\right)$ are the coordinates for the steer wheel. These equations establish that lateral displacement is null. Now, using the constraints given for a rigid body, we have that

$$
x_{f}=x+L \cos \theta, \quad y_{f}=y+L \sin \theta .
$$

Substituting into the first equation and defining the radius of the curve that the vehicle describes as $R=L\left(\operatorname{tg}^{-1} \phi\right)$, the cinematic model for a car-like vehicle is

$$
\left(\begin{array}{c}
\dot{x} \\
\dot{y} \\
\dot{\theta} \\
\dot{\phi}
\end{array}\right)=\left(\begin{array}{c}
\cos \theta \\
\sin \theta \\
\frac{1}{L} \operatorname{tg} \phi \\
0
\end{array}\right) v_{1}+\left(\begin{array}{l}
0 \\
0 \\
0 \\
1
\end{array}\right) v_{2}
$$

where $v_{1}$ and $v_{2}$ are the velocities for the vehicle and the direction, respectively. A more complete model should include rotation angles for each wheel, or take into account the possible deformation of the wheels; however, the simple proposed model has the essential elements for the analysis, and should be enough for control purposes [21].

\section{AV's POSITION ESTIMATE UNCERTAINTY}

We propose using the position-estimation equations of the cinematic model to obtain the covariance matrix expressing the uncertainty for the odometry estimated position. This uncertainty estimation takes directly into account the physical structure of the vehicle, which is translated into the control variables being manipulated and sensed. For the case at hand, the only inputs to the model, used to obtain the $(x, y, \theta)$ variables required to compute the position of the vehicle, are the two measures being obtained for internal sensors: the velocity, translated into the instantaneous displacement $\Delta d_{t}^{\text {od }}$; and the instantaneous steering angle $\phi_{t}$. Hence, a closed mathematical form for such a matrix will be obtained. For time $t$, given a position estimate $P_{t}$, we have an associated uncertainty in the estimation given by $\operatorname{Cov}\left[P_{t}\right]$.

The position estimate from odometry at time $t$ is computed as $P_{t}^{\text {od }}=$ $P_{t-1}+\Delta P_{t}^{\text {od }}$, corresponding to the sum of the robot position in a previous time $t-1$, plus the increments in position measured from the 
odometry system and computed using the equations from the cinematic model

$$
\Delta P_{t}^{\text {od }}=\left(\begin{array}{c}
\Delta x_{t}^{\text {od }} \\
\Delta y_{t}^{\text {od }} \\
\Delta \theta_{t}^{\text {od }}
\end{array}\right)=\left(\begin{array}{c}
\Delta \hat{d}_{t}^{\text {od }} \cos \left(\theta_{t-1}+\frac{\Delta \theta_{t}}{2}\right) \\
\Delta \hat{d}_{t}^{\text {od }} \sin \left(\theta_{t-1}+\frac{\Delta \theta_{t}}{2}\right) \\
\frac{\Delta \hat{d}_{t}^{\text {od }}}{L} \operatorname{tg}\left(\hat{\phi}_{t}\right)
\end{array}\right)
$$

Measures obtained from both odometers and steer-angle sensors are not error-free. In order to compute the uncertainty in the position given by the odometry system, $\operatorname{Cov}\left[P_{t}^{\text {od }}\right]$, we consider that those errors follow a normal distribution ${ }^{1}$

$$
\begin{aligned}
\Delta \hat{d}_{t}^{\text {od }} & =\Delta d_{t}^{\text {od }}+\varepsilon^{\text {od }}, \quad \text { where } \varepsilon^{\text {od }} \approx N\left(0, \sigma_{\text {od }}^{2}\right) \\
\hat{\phi}_{t} & =\phi_{t}+\varepsilon^{\phi}, \quad \text { where } \varepsilon^{\phi} \approx N\left(0, \sigma_{\phi}^{2}\right) .
\end{aligned}
$$

So the uncertainty in the position $P_{t}^{\text {od }}$ is given by

$$
\begin{aligned}
\operatorname{Cov}\left[P_{t}^{\text {od }}\right] & =\operatorname{Cov}\left[P_{t-1}+\Delta P_{t}^{\text {od }}\right] \\
& =\operatorname{Cov}\left[P_{t-1}\right]+\operatorname{Cov}\left[\Delta P_{t}^{\text {od }}\right]+2 \operatorname{Cov}\left[P_{t-1}, \Delta P_{t}^{\text {od }}\right] .
\end{aligned}
$$

In order to solve (3) for our vehicle, it is necessary to calculate each of the elements of the involved matrices. The first element in (4) is calculated as follows:

$$
\begin{aligned}
E & {\left[\Delta x_{t}^{\text {od }} \Delta x_{t}^{\text {od }}\right] } \\
& =E\left[\Delta \hat{d}_{t}^{\text {od }} \cos \left(\theta_{t-1}+\frac{\Delta \theta_{t}}{2}\right) \Delta \hat{d}_{t}^{\text {od }} \cos \left(\theta_{t-1}+\frac{\Delta \theta_{t}}{2}\right)\right] \\
& =E\left[\left(\Delta \hat{d}_{t}^{\text {od }}\right)^{2} \cos ^{2}\left(\theta_{t-1}+\frac{\Delta \theta_{t}}{2}\right)\right] \\
& =\frac{1}{2} E\left[\left(\Delta \hat{d}_{t}^{\text {od }}\right)^{2}\left(1+\cos \left(2 \theta_{t-1}\right)-\sin \left(2 \theta_{t-1}\right) \Delta \theta_{t}\right)\right]
\end{aligned}
$$

where the following equivalences have been used:

$$
\begin{aligned}
\cos ^{2}(\alpha) & =\frac{1}{2}(1+\cos (2 \alpha)) \\
\cos (\alpha \pm \beta) & =\cos \alpha \cos \beta \mp \sin \alpha \sin \beta
\end{aligned}
$$

We considered also, in order to simplify the presented mathematical development and without loss of generality of the given method, the assumption that $\Delta \theta_{t}$ is small between two consecutive measures. Such an assumption is guaranteed, because our AV moves at low speeds, and a high sampling frequency for sensors is used. Hence, the mathematical development presented in this paper may not be valid for vehicles moving at high speed. Should we need to calculate the position uncertainty of a high-speed vehicle, we would only need to reformulate the given mathematical expressions without the limitation of $\Delta \theta_{t}$ small. Going on the calculation

$$
\begin{aligned}
E\left[\Delta x_{t}^{\text {od }} \Delta x_{t}^{\text {od }}\right]= & \cdots \\
= & \frac{1}{2} E\left[\left(\Delta \hat{d}_{t}^{\text {od }}\right)^{2}\right] \\
& +\frac{1}{2} E\left[\left(\Delta \hat{d}_{t}^{\text {od }}\right)^{2} \cos \left(2 \theta_{t-1}\right)\right] \\
& -\frac{1}{2} E\left[\left(\Delta \hat{d}_{t}^{\text {od }}\right)^{2} \sin \left(2 \theta_{t-1}\right) \Delta \theta_{t}\right]
\end{aligned}
$$

${ }^{1}$ In fact, to compute errors from odometers, we considered left and right odometers separately, obtaining $\sigma_{\mathrm{od}, r}^{2}, \sigma_{\mathrm{od}, l}^{2}$, and computing $4 \sigma_{\mathrm{od}}^{2}=\sigma_{\mathrm{od}, r}^{2}+$ $\sigma_{\text {od }, l}^{2}$.
Particularizing each term of the previous expression

$$
\begin{aligned}
\frac{1}{2} E & {\left[\left(\Delta \hat{d}_{t}^{\text {od }}\right)^{2}\right] } \\
= & \frac{1}{2} E\left[\left(\Delta d_{t}^{\text {od }}+\varepsilon^{\text {od }}\right)\left(\Delta d_{t}^{\text {od }}+\varepsilon^{\text {od }}\right)\right] \\
= & \frac{1}{2}\left(\left(\Delta d_{t}^{\text {od }}\right)^{2}+\sigma_{\text {od }}^{2}\right) \\
\frac{1}{2} E & {\left[\left(\Delta \hat{d}_{t}^{\text {od }}\right)^{2} \cos \left(2 \theta_{t-1}\right)\right] } \\
= & \left(\left(\Delta d_{t}^{\text {od }}\right)^{2}+\sigma_{\text {od }}^{2}\right)\left(\cos ^{2}\left(\theta_{t-1}\right)-\frac{1}{2}\right) \\
\frac{1}{2} E & {\left[\left(\Delta \hat{d}_{t}^{\text {od }}\right)^{2} \sin \left(2 \theta_{t-1}\right) \Delta \theta_{t}\right] } \\
= & \sin \left(\theta_{t-1}\right) \cos \left(\theta_{t-1}\right) E\left[\left(\Delta \hat{d}_{t}^{\text {od }}\right)^{2} \Delta \theta_{t}\right] \\
= & \frac{1}{L} \sin \left(\theta_{t-1}\right) \cos \left(\theta_{t-1}\right) E\left[\left(\Delta \hat{d}_{t}^{\text {od }}\right)^{3} \operatorname{tg} \hat{\phi}_{t}\right] \\
= & \frac{1}{L} \sin \left(\theta_{t-1}\right) \cos \left(\theta_{t-1}\right) E\left[\left(\Delta \hat{d}_{t}^{\text {od }}\right)^{3}\right] E\left[t \hat{\phi}_{t}\right] \\
= & \left(\left(\Delta d_{t}^{\text {od }}\right)^{3}+3 \Delta d_{t}^{\text {od }} \sigma_{\text {od }}^{2}\right) \operatorname{tg}\left(\phi_{t}+\varepsilon_{\max }^{\phi}\right)
\end{aligned}
$$

where we considered independence between sensor measures, and physical considerations on the steer angle measurement have been taken into account in order to solve $E\left[\operatorname{tg} \hat{\phi}_{t}\right]$.

After computing all the expectances, a closed-form expression for $\operatorname{Cov}\left(\Delta P_{t}^{\text {od }}\right)$ is obtained, as the rest of two symmetric $3 \times 3$ matrices, $E\left[\Delta P_{t}^{\text {od }} \Delta P_{t}^{T^{\text {od }}}\right]-E\left[\Delta P_{t}^{\text {od }}\right] E\left[\Delta P_{t}^{T^{\text {od }}}\right]$. We define the constants $k_{1}$ and $k_{2}$ as

$$
k_{1}=\left(\Delta d_{t}^{\mathrm{od}}\right)^{2}+\sigma_{\mathrm{od}}^{2}, \quad k_{2}=\frac{1}{L}\left(\left(\Delta d_{t}^{\mathrm{od}}\right)^{3}+3 \Delta d_{t}+\sigma_{\mathrm{od}}^{2}\right) .
$$

Elements for the first matrix, given in (3), are

$$
\begin{aligned}
c_{11}= & k_{1} \cos ^{2}\left(\theta_{t-1}\right)-k_{2} \sin \left(\theta_{t-1}\right) \cos \left(\theta_{t-1}\right) \operatorname{tg}\left(\phi_{t}+\varepsilon_{\max }^{\phi}\right) \\
c_{12}= & c_{21}=\frac{k_{1}}{2} \sin \left(2 \theta_{t-1}\right)+\frac{k_{2}}{2} \cos \left(2 \theta_{t-1}\right) \operatorname{tg}\left(\phi_{t}+\varepsilon_{\max }^{\phi}\right) \\
c_{13}= & c_{31}=\frac{k_{1}}{L} \cos \left(\theta_{t-1}\right) \operatorname{tg}\left(\phi_{t}+\varepsilon_{\max }^{\phi}\right) \\
& -\frac{k_{2}}{2 L} \sin \left(\theta_{t-1}\right) t^{2}\left(\phi_{t}+\varepsilon_{\max }^{\phi}\right) \\
c_{22}= & \frac{k_{1}}{2}\left(1-\cos \left(2 \theta_{t-1}\right)\right)+\frac{k_{2}}{2} \sin \left(2 \theta_{t-1}\right) \operatorname{tg}\left(\phi_{t}+\varepsilon_{\max }^{\phi}\right) \\
c_{23}= & c_{32}=\frac{k_{1}}{L} \sin \left(\theta_{t-1}\right) \operatorname{tg}\left(\phi_{t}+\varepsilon_{\max }^{\phi}\right) \\
& +\frac{k_{2}}{2 L} \cos \left(\theta_{t-1}\right) g^{2}\left(\phi_{t}+\varepsilon_{\max }^{\phi}\right) \\
c_{33}= & \frac{k_{1}}{L^{2}} t^{2}\left(\phi_{t}+\varepsilon_{\max }^{\phi}\right) .
\end{aligned}
$$

A complete development of all the elements for matrix (4) is given in the Appendix. The second matrix, (5), is computed from the vector product $E\left[\Delta P_{t}^{\text {od }}\right] E\left[\Delta P_{t}^{T^{\text {od }}}\right]$, where

$$
E\left[\Delta P_{t}^{\text {od }}\right]=\left(\begin{array}{c}
\Delta d_{t}^{\text {od }} \cos \left(\theta_{t-1}\right)-\frac{k_{1}}{2 L} \sin \left(\theta_{t-1}\right) \operatorname{tg}\left(\phi_{t}+\varepsilon_{\max }^{\phi}\right) \\
\Delta d_{t}^{\text {od }} \sin \left(\theta_{t-1}\right)-\frac{k_{1}}{2 L} \cos \left(\theta_{t-1}\right) \operatorname{tg}\left(\phi_{t}+\varepsilon_{\max }^{\phi}\right) \\
\frac{\Delta d_{t}^{\text {od }}}{L} t g\left(\phi_{t}+\varepsilon_{\max }^{\phi}\right)
\end{array}\right) .
$$




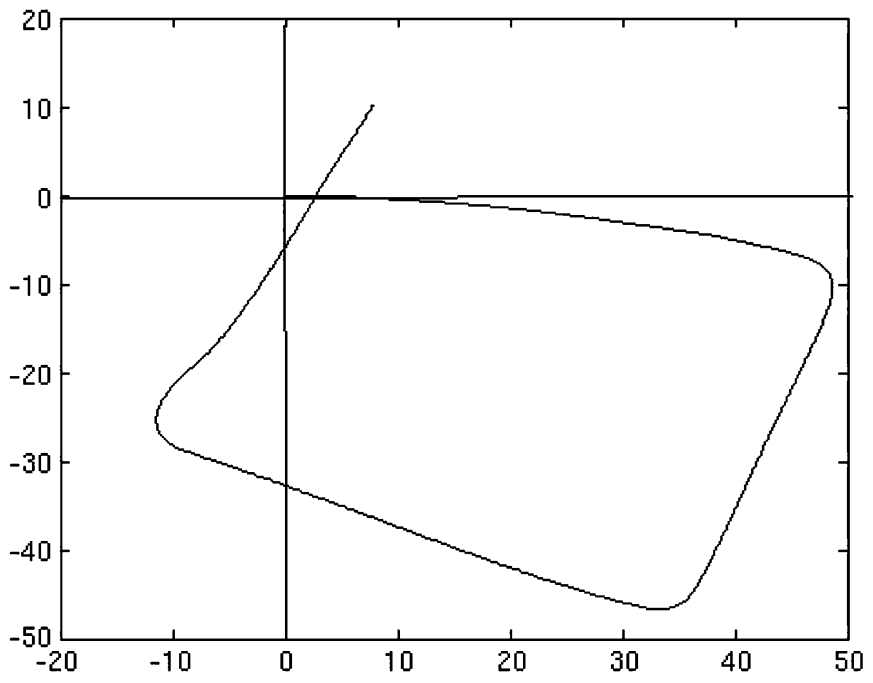

Fig. 3. Odometry data obtained for the run path. The proposed path starts at the origin $(0,0)$, advancing from left to right in the graph to complete a round trip.

The full expression for this second matrix is not included, due to the length of the involved expressions. It can be easily and directly derived from multiplying $E\left[\Delta P_{t}^{\text {od }}\right] E\left[\Delta P_{t}^{T^{\text {od }}}\right]$. Finally, the closed-form expression for the uncertainty in the odometry position estimate will be given by subtracting the two recently computed matrices $E\left[\Delta P_{t}^{\text {od }} \Delta P_{t}^{T^{\text {od }}}\right]-E\left[\Delta P_{t}^{\text {od }}\right] E\left[\Delta P_{t}^{T^{\text {od }}}\right]$. The mathematical expression for the final matrix is not meaningful by itself, it being much more practical to use a computer code that computes both matrices separately and then subtracts them.

\section{ASSESSMENT OF RESUlTS}

To assess the effectiveness of the proposed method to compute the uncertainty associated with the odometry position estimate of a mobile vehicle, an experiment was designed and run using the AV described in Section III. A $50 \times 40$-m rectangular path, marked on the floor of the ITESM parking lot, was followed while manually driving the described AV. Data from internal sensors (encoders and steer angle) were gathered during the run. ${ }^{2}$ Fig. 3 shows the obtained odometry data for coordinates $(x, y)$. Then, the uncertainty of the odometry position estimates obtained with both an EKF and the proposed method was compared. Figs. 4 and 5 show the obtained covariance, denoting the uncertainty of the position estimates, for the $x$ and $y$ axes, using the proposed formulation and an EKF, respectively.

It can be seen from Figs. 4 and 5 that the obtained uncertainty for coordinates $x$ and $y$ using the method presented in this paper (about $10 \mathrm{~m}$ ) is lower than that obtained with an EKF (about $17 \mathrm{~m}$ ). It turns out that the real measured position error for the used platform in the run experiment is about $10 \mathrm{~m}$ (which means a covariance uncertainty of about 100, as our method states).

\section{CONCLUSIONS AND FUture WORK}

The position of a robot or an AV in a two-dimensional space can be represented by the three components vector $(x, y, \theta)$ where $x$ and $y$ represent the position coordinates of the robot on the space, and $\theta$ its orientation. Thus, the robot position at time $t$ is denoted $P_{t}=$ $\left(x_{t}, y_{t}, \theta_{t}\right)^{T}$. There is always an error associated with the movement of the autonomous platform. The imprecision in the position estimate

${ }^{2}$ Maximum velocity was $8 \mathrm{~km} / \mathrm{h}$. Sampling time interval was $10 \mathrm{~Hz}$.

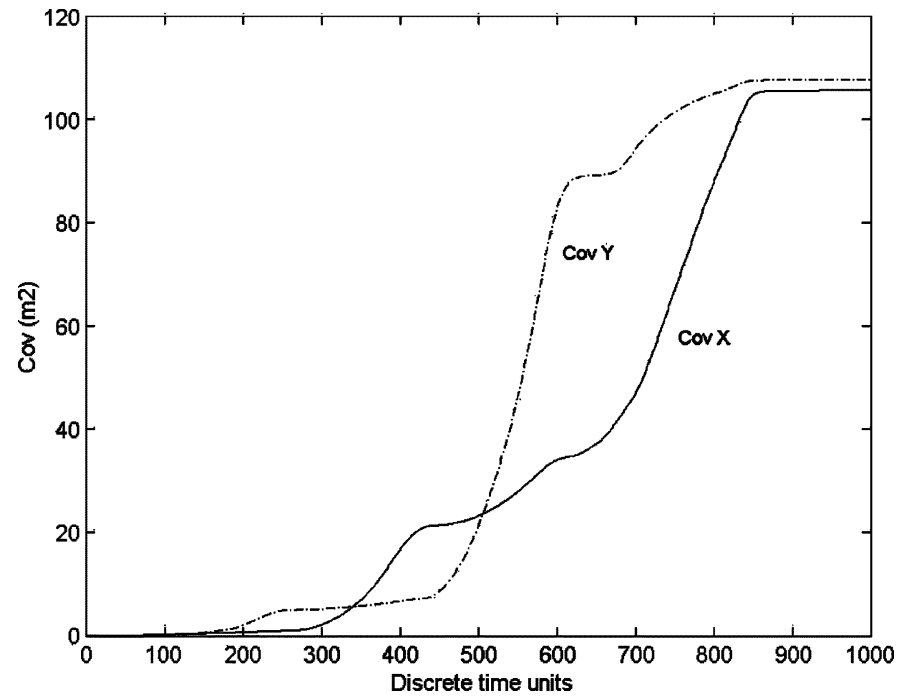

Fig. 4. Estimated position uncertainty for coordinates $x, y$ using the presented formulation.

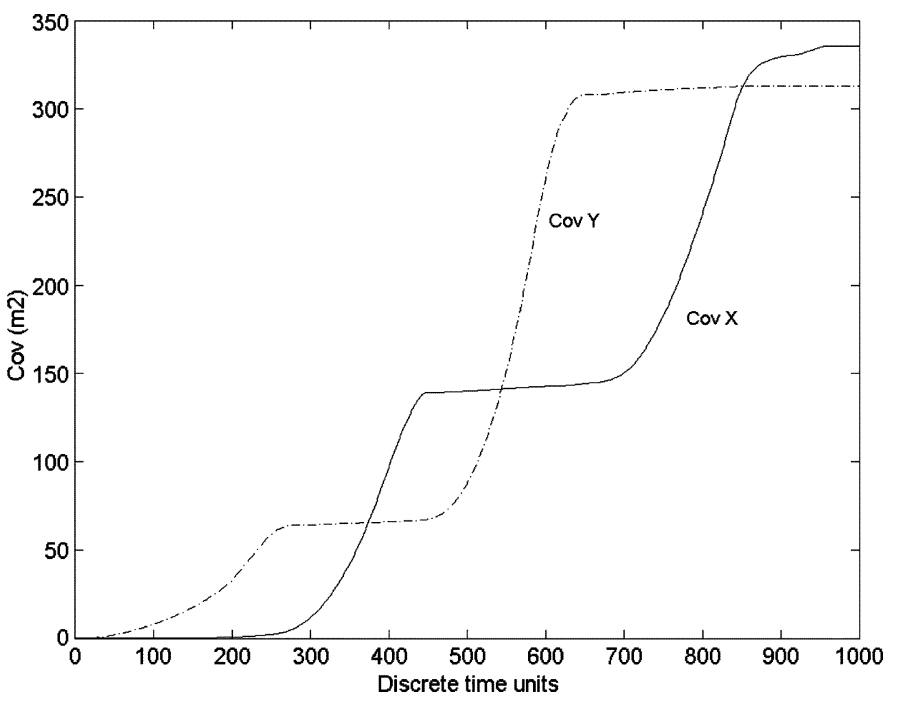

Fig. 5. Estimated position uncertainty for coordinates $x, y$ from an EKF. Note the linearity and dependency to the orientation in the measurement, in regard to the axis, given by the value steps.

is due to errors in the sensory system used to determinate such estimations, as well as unmodeled factors in the vehicle model. These errors are normally estimated from data derived from experimentation and then integrated in algorithms, as for example, Kalman filtering, which are highly sensitive to the obtained parameters.

We have proposed in this paper a way to obtain a closed-form expression for the position uncertainty, by means of a covariance matrix, when position estimates are being obtained using internal sensors. The proposed method is valid for any platform or set of sensors. We then particularize the formulation in order to obtain a closed-form expression for the covariance matrix representing the measure of position uncertainty for a given kind of $\mathrm{AV}$, consisting of a nonholonomic Ackerman architecture. For the platform used in this study, it is important to note that the instantaneous control variables are $\Delta d_{t}^{\text {od }}$ and $\phi_{t}$, the displacement in function of time and the steering angle, respectively; these are the sole variables that the internal system can manipulate and sense.

Even if a lot of research and experiments are left for the near future, the initial experimental evaluation demonstrates that the proposed 
method computes the uncertainty in position with higher accuracy than the standard EKF method. At the same time, the obtained data suggest that the proposed method is independent of the orientation of the vehicle in regard to the axis, in contrast to the EKF method, where the uncertainty grows in regard to the axis to which the vehicle is oriented. The authors are actually working on the integration of such a matrix computation into a probabilistic frame (errors in the positioning sensors can be assumed to be random, and can be modeled by a parametric probability distribution) that allows performing data fusion from different positioning systems; concretely, work is being done on the integration of odometry and GPS measures. We hope that the formulation presented in this paper will help to reduce errors when estimating the position of the vehicle using different sources of data.

\section{APPENDIX}

A complete derivation of the terms of the first matrix expressed in (4) is given here. The first element in (4) was calculated in Section IV. Taking into account that the matrix is symmetric, and $\Delta \theta_{t}$ is small between two consecutive measures, the rest of the elements are calculated as follows:

$$
\begin{aligned}
\mathrm{c} 12 & =\mathrm{c} 21=E\left[\Delta x_{t}^{\text {od }} \Delta y_{t}^{\text {od }^{\mathrm{T}}}\right] \\
& =E\left[\Delta \hat{d}_{t}^{\text {od }} \cos \left(\theta_{t-1}+\frac{\Delta \theta_{t}}{2}\right) \Delta \hat{d}_{t}^{\text {od }} \sin \left(\theta_{t-1}+\frac{\Delta \theta_{t}}{2}\right)\right] \\
& =E\left[\frac{1}{2}\left(\Delta \hat{d}_{t}^{\text {od }}\right)^{2} \sin \left(2 \theta_{t-1}+\Delta \theta_{t}\right)\right] \\
& =\frac{1}{2} E\left[( \Delta \hat { d } _ { t } ^ { \text { od } } ) ^ { 2 } \left(\sin \left(2 \theta_{t-1}\right) \cos \left(\Delta \theta_{t}\right)\right.\right. \\
& \left.\left.\quad+\cos \left(2 \theta_{t-1}\right) \sin \left(\Delta \theta_{t}\right)\right)\right] \\
& =\frac{1}{2} E\left[\left(\Delta \hat{d}_{t}^{\text {od }}\right)^{2}\left(\sin \left(2 \theta_{t-1}\right)+\cos \left(2 \theta_{t-1}\right) \Delta \theta_{t}\right)\right] .
\end{aligned}
$$

In the last three equalities, the following equivalences and assumptions have been used:

$$
\begin{aligned}
\sin (2 \alpha) & =2 \sin (\alpha) \cos (\alpha) \\
\sin (\alpha \pm \beta) & =\sin \alpha \cos \beta \pm \cos \alpha \sin \beta
\end{aligned}
$$

Going on the calculation and using some of the results given in Section IV when computing $\mathrm{c} 11$

$$
\begin{aligned}
E[ & \left.\Delta x_{t}^{\text {od }} \Delta y_{t}^{\text {od }}{ }^{\mathrm{T}}\right] \\
= & \frac{1}{2} E\left[\left(\Delta \hat{d}_{t}^{\text {od }}\right)^{2} \sin \left(2 \theta_{t-1}\right)\right] \\
& +\frac{1}{2} E\left[\left(\Delta \hat{d}_{t}^{\text {od }}\right)^{2} \cos \left(2 \theta_{t-1}\right) \Delta \theta_{t}\right] \\
= & \cdots=\frac{k_{1}}{2} \sin \left(2 \theta_{t-1}\right)+\frac{k_{2}}{2} \cos \left(2 \theta_{t-1}\right) \operatorname{tg}\left(\phi_{t}+\varepsilon_{\max }^{\phi}\right) \\
= & c 12=c 21 . \\
\mathrm{c} 13= & \mathrm{c} 31=E\left[\Delta x_{t}^{\text {od }} \Delta \theta_{t}^{\text {od }}\right] \\
= & E\left[\Delta \hat{d}_{t}^{\text {od }} \cos \left(\theta_{t-1}+\frac{\Delta \theta_{t}}{2}\right) \frac{\Delta \hat{d}_{t}^{\text {od }}}{L} \operatorname{tg}\left(\hat{\phi}_{t}\right)\right] \\
= & \frac{1}{L} E\left[( \Delta \hat { d } _ { t } ^ { \text { od } } ) ^ { 2 } \operatorname { t g } ( \hat { \phi } _ { t } ) \left(\cos \left(\theta_{t-1}\right) \cos \left(\frac{\Delta \theta_{t}}{2}\right)\right.\right.
\end{aligned}
$$

$$
\begin{aligned}
= & \frac{1}{L} \cos \left(\theta_{t-1}\right) E\left[\left(\Delta \hat{d}_{t}^{\text {od }}\right)^{2} \operatorname{tg}\left(\hat{\phi}_{t}\right)\right] \\
& -\frac{1}{2 L} \sin \left(\theta_{t-1}\right) E\left[\left(\Delta \hat{d}_{t}^{\text {od }}\right)^{2} \Delta \theta_{t} \operatorname{tg}\left(\hat{\phi}_{t}\right)\right] \\
= & \frac{1}{L} \cos \left(\theta_{t-1}\right) E\left[\left(\Delta \hat{d}_{t}^{\text {od }}\right)^{2}\right] E\left[\operatorname{tg}\left(\hat{\phi}_{t}\right)\right] \\
& -\frac{1}{2 L} \sin \left(\theta_{t-1}\right) E\left[\left(\Delta \hat{d}_{t}^{\text {od }}\right)^{2} \frac{\Delta \hat{d}_{t}^{\text {od }}}{L}\right] E\left[\operatorname{tg}\left(\hat{\phi}_{t}\right) \operatorname{tg}\left(\hat{\phi}_{t}\right)\right] \\
= & \cdots=\frac{k_{1}}{L} \cos \left(\theta_{t-1}\right) \operatorname{tg}\left(\phi_{t}+\varepsilon_{\max }^{\phi}\right) \\
& \quad-\frac{k_{2}}{2 L} \sin \left(\theta_{t-1}\right) \operatorname{tg}^{2}\left(\phi_{t}+\varepsilon_{\max }^{\phi}\right) .
\end{aligned}
$$

The following equivalences have been used:

$$
\cos (\alpha \pm \beta)=\cos \alpha \cos \beta \mp \sin \alpha \sin \beta
$$

$$
\begin{aligned}
& \mathrm{c} 22= E\left[\Delta y_{t}^{\text {od }} \Delta y_{t}^{\text {od }}\right] \\
&= E\left[\Delta \hat{d}_{t}^{\text {od }} \sin \left(\theta_{t-1}+\frac{\Delta \theta_{t}}{2}\right) \Delta \hat{d}_{t}^{\text {od }} \sin \left(\theta_{t-1}+\frac{\Delta \theta_{t}}{2}\right)\right] \\
&= \frac{1}{2} E\left[\left(\Delta \hat{d}_{t}^{\text {od }}\right)^{2}\left(1-\cos \left(2 \theta_{t-1}+\Delta \theta_{t}\right)\right)\right] \\
&= \frac{1}{2} E\left[\left(\Delta \hat{d}_{t}^{\text {od }}\right)^{2}\left(1-\cos \left(2 \theta_{t-1}\right)+\Delta \theta_{t} \sin \left(2 \theta_{t-1}\right)\right)\right] \\
&= \frac{1}{2} E\left[\left(\Delta \hat{d}_{t}^{\text {od }}\right)^{2}\right]-\frac{1}{2} \cos \left(2 \theta_{t-1}\right) E\left[\left(\Delta \hat{d}_{t}^{\text {od }}\right)^{2}\right] \\
&+\frac{1}{2 L} \sin \left(2 \theta_{t-1}\right) E\left[\left(\Delta \hat{d}_{t}^{\text {od }}\right)^{3}\right] E\left[\operatorname{tg}\left(\hat{\phi}_{t}\right)\right] \\
&= \cdots=\frac{k_{1}}{2}\left(1-\cos \left(2 \theta_{t-1}\right)\right) \\
& \quad+\frac{k_{2}}{2} \sin \left(2 \theta_{t-1}\right) \operatorname{tg}\left(\phi_{t}+\varepsilon_{\max }^{\phi}\right)
\end{aligned}
$$

where the following equivalences have been used:

$$
\sin ^{2} \alpha=\frac{1}{2}(1-\cos 2 \alpha), \cos (\alpha \pm \beta)=\cos \alpha \cos \beta \mp \sin \alpha \sin \beta
$$

$$
\begin{aligned}
c 23= & c 32=E\left[\Delta y_{t}^{\text {od }} \Delta \theta_{t}^{\text {od }}{ }^{\mathrm{T}}\right] \\
= & E\left[\Delta \hat{d}_{t}^{\text {od }} \sin \left(\theta_{t-1}+\frac{\Delta \theta_{t}}{2}\right) \frac{\Delta \hat{d}_{t}^{\text {od }}}{L} t g\left(\hat{\phi}_{t}\right)\right] \\
= & \frac{1}{L} E\left[( \Delta \hat { d } _ { t } ^ { \text { od } } ) ^ { 2 } \left(\sin \left(\theta_{t-1}\right) \cos \left(\frac{\Delta \theta_{t}}{2}\right)\right.\right. \\
& \left.\left.\quad+\cos \left(\theta_{t-1}\right) \sin \left(\frac{\Delta \theta_{t}}{2}\right)\right) \operatorname{tg} \hat{\phi}_{t}\right] \\
= & \frac{1}{L} E\left[\left(\Delta \hat{d}_{t}^{\text {od }}\right)^{2}\left(\sin \left(\theta_{t-1}\right)+\cos \left(\theta_{t-1}\right) \frac{\Delta \theta_{t}}{2}\right) \operatorname{tg} \hat{\phi}_{t}\right] \\
= & \frac{1}{L} \sin \left(\theta_{t-1}\right) E\left[\left(\Delta \hat{d}_{t}^{\text {od }}\right)^{2}\right] E\left[\operatorname{tg} \hat{\phi}_{t}\right] \\
& +\frac{1}{2 L^{2}} \cos \left(\theta_{t-1}\right) E\left[\left(\Delta \hat{d}_{t}^{\text {od }}\right)^{3}\right] E\left[g^{2} \hat{\phi}_{t}\right] \\
= & \frac{k_{1}}{L} \sin \left(\theta_{t-1}\right) \operatorname{tg}\left(\phi_{t}+\varepsilon_{\max }^{\phi}\right) \\
& +\frac{k_{2}}{2 L} \cos \left(\theta_{t-1}\right) \operatorname{tg}\left(\phi_{t}+\varepsilon_{\max }^{\phi}\right) .
\end{aligned}
$$


The following equivalences and assumptions have been used:

$$
\begin{aligned}
\sin (\alpha \pm \beta)=\sin \alpha \cos \beta \pm \cos \alpha \sin \beta . \\
\cos =E\left[\Delta \theta_{t}^{\text {od }} \Delta \theta_{t}^{\text {od }}\right] \\
=E\left[\frac{\Delta \hat{d}_{t}^{\text {od }}}{L} \operatorname{tg}\left(\hat{\phi}_{t}\right) \frac{\Delta \hat{d}_{t}^{\text {od }}}{L} \operatorname{tg}\left(\hat{\phi}_{t}\right)\right] \\
=\frac{1}{L^{2}} E\left[\left(\Delta \hat{d}_{t}^{\text {od }}\right)^{2}\right] E\left[\operatorname{tg}^{2} \hat{\phi}_{t}\right] \\
=\frac{k_{1}}{L^{2}} t^{2}\left(\phi_{t}+\varepsilon_{\max }^{\phi}\right) .
\end{aligned}
$$

\section{ACKNOWLEDGMENT}

One of the authors, J. M. Mirats Tur, wishes to thank the Department of Computer Science, Instituto Tecnológico de Monterrey (ITESM) for holding him as an Invited Professor during 2004. This work was possible thanks to the invitation from J. Nolazco, Director of the department.

\section{REFERENCES}

[1] M. A. Salichs, J. M. Arminol, J. M. Moreno, and A. de la Escalera, "Localization system for mobile robots in indoor environments," Integr. Computer-Aided Eng., vol. 6, no. 4, pp. 303-318, 1999.

[2] J. Howell and B. R. Donald, "Practical mobile robot self-localization," in Proc. IEEE Int. Conf. Robot. Autom., San Francisco, CA, Apr. 2000, pp. 3485-3492.

[3] R. G. Brown and B. R. Donald, "Mobile robot self-localization without explicit landmarks," Algorithmica, vol. 26, no. 3/4, pp. $515-559,2000$.

[4] S. Thrun, "Finding landmarks for mobile robot navigation," in Proc. IEEE Int. Conf. Robot. Autom., 1998, pp. 958-963.

[5] J. R. Asensio, J. M. Montiel, and L. Montano, "Goal directed reactive robot navigation with relocation using laser and vision," in Proc. IEEE Int. Conf. Robot. Autom., May 1999, pp. 2905-2910.

[6] H. Gonzalez-Banos and J. C. Latombe, "Navigation strategies for exploring indoor environments," Int. J. Robot. Res., vol. 21, no. 10-11, pp. 829-848, Oct.-Nov. 2002.

[7] R. Thrapp and C. Westbrook, "Robust localization algorithms for an autonomous campus tour guide," in Proc. IEEE Int. Conf. Robot. Autom., Seoul, Korea, May 2001, pp. 2065-2071.

[8] T. Pilarsky, M. Happold, H. Pangels, M. Ollis, K. Fitzpatrick, and A. Stentz, "The demeter system for automated harvesting," Auton. Robots, vol. 13, pp. 9-20, 2002.

[9] S. Scheding, G. Dissanayake, E. M. Nebot, and H. Durrant-Whyte, "An experiment in autonomous navigation of an underground mining vehicle," IEEE Trans. Robot. Autom., vol. 15, no. 1, pp. 85-95, Feb. 1999.

[10] H. Chung, L. Ojeda, and J. Borenstein, "Sensor fusion for mobile robot dead reckoning with a precision-calibrated fiber optic gyroscope," in Proc. IEEE Int. Conf. Robot. Autom., Seoul, Korea, May 2001, pp. 3588-3593.

[11] S. Majumder, S. Scheding, and H. F. Durrant-Whyte, "Multi sensor data fusion for underwater navigation," Robot. Auton. Syst., vol. 35, no. 1, pp. 97-108, 2001.

[12] R. A. Brooks, "Visual map making for a mobile robot," in Proc. IEEE Int. Conf. Robot. Autom., St. Louis, MO, 1985, pp. 824-829.

[13] R. Chatila and J. P. Laumond, "Position referencing and consistent world modeling for mobile robots," in Proc. IEEE Int. Conf. Robot. Autom., St. Louis, MO, 1985, pp. 138-145.

[14] R. C. Smith and P. Cheeseman, "On the representation and estimation of spatial uncertainty," Int. J. Robot. Res., vol. 5, pp. 55-68, 1987.

[15] C. M. Wang, "Location estimation and uncertainty analysis for mobile robots," in Proc. IEEE Int. Conf. Robot. Autom., 1988, pp. 1230-1235.

[16] A. Kelly, "General solution for linearized systematic error propagation in vehicle odometry," in Proc. Int. Conf. Intell. Robots Syst., Maui, HI, Oct.-Nov. 2001, pp. 1938-1945.
[17] A. Pozo-Ruz, "Sistema sensorial para localización de vehículos en exteriores," Ph.D. dissertation (in Spanish), Dept. Elect. Eng., Univ. Málaga, Málaga, Spain, 2001.

[18] K. S. Chong and L. Kleeman, "Accurate odometry and error modeling for a mobile robot," in Proc. IEEE Int. Conf. Robot. Autom., 1997, pp. 2783-2788.

[19] A. Martinelli, "The odometry error of a mobile robot with a synchronous drive system," IEEE Trans. Robot. Autom., vol. 18, no. 3, pp. 399-405, Jun. 2002.

[20] G. Palacios, "Control de dirección de un vehículo autónomo," M.S. thesis (in Spanish), Inst. Tecnológico de Monterrey, Monterrey, México, 2000.

[21] J. P. Laumond, "Robot motion planning and control," Lab. d'Analyze et d'Architecture des Systèmes, Toulouse, France, LAAS Rep. 97438 , 1998.

\section{A Stabilizing Receding Horizon Regulator for Nonholonomic Mobile Robots}

\author{
Dongbing $\mathrm{Gu}$ and Huosheng $\mathrm{Hu}$
}

\begin{abstract}
This paper presents a receding horizon (RH) controller used for regulating a nonholonomic mobile robot. The RH control stability is guaranteed by adding a terminal-state penalty to the cost function and a terminal-state region to optimization constraints. A suboptimal solution to the optimization problem is sufficient to achieve stability. A new terminalstate penalty and its corresponding terminal-state constraints are found. Implementation and simulation results are provided to verify the proposed control strategy.
\end{abstract}

Index Terms-Model predictive control (MPC), nonholonomic mobile robots, receding horizon $(\mathrm{RH})$ control.

\section{INTRODUCTION}

Regulating or stabilizing nonholonomic mobile robots is to move the robot toward a goal pose from any initial pose. The linearized models of nonholonomic systems are not controllable, and continuous time-invariant feedback control does not exist in the regulation problem [6]. So far, a number of control strategies have been proposed to handle this problem, including Lyapunov control [1], [4], [7], [13], nonlinear geometric control [5], [20], [21], [23], [26], discontinuous control [4], piecewise-continuous feedback control [7], smooth time-varying control [20], [26], and dynamic feedback linearization [21].

Receding horizon (RH) or model predictive control (MPC) is one of the frequently applied advanced control techniques in industry, which is designed to handle optimization problems (OPs) with constraints. Due to the use of a predictive control horizon, the control stability becomes one of the main problems. It was shown that using an infinite receding horizon can guarantee RH control stability for even nonlinear systems [17], but it is computationally intractable in practice. For a finite receding horizon, it was proved that the stability can be guaranteed by forcing the terminal state to equal zero [25]. However, the terminal equality constraint is time consuming. Further work shows that the terminal-state equality constraint can be relaxed as a terminal-state inequality, i.e., a terminal-state region, by adding a terminal-state penalty

Manuscript received July 20, 2004; revised November 8, 2004. This paper was recommended for publication by Associate Editor G. Oriolo and Editor H. Arai upon evaluation of the reviewers' comments.

The authors are with the University of Essex, Colchester CO4 3SQ, U.K. (e-mail:dgu@essex.ac.uk; hhu@essex.ac.uk).

Digital Object Identifier 10.1109/TRO.2005.851357 